\title{
A Revised Model for Magneto Convection in Binary Nanofluids
}

\author{
Jyoti Sharma \\ University Institute of Engineering \& Technology \\ Panjab University, Chandigarh, India \\ Corresponding author: jyoti.maths@gmail.com
}

Urvashi Gupta

Dr. S. S. Bhatnagar University Institute of Chemical Engineering and Technology Panjab University, Chandigarh, India

\author{
Shushant Shukla \\ Energy Research Centre \\ Panjab University, Chandigarh, India
}

(Received February 21, 2018; Accepted August 11, 2018)

\begin{abstract}
The paper presents double-diffusive nanofluid convection under magnetic field using more realistic revised model in which boundaries are assumed to have zero nanoparticle flux. The nanofluid layer includes the nano scale effects (Brownian motion and thermophoresis) and solutal effects (Dufour and Soret). Impact of different parameters is analyzed using normal mode technique and interpreted graphically with the help of the software Mathematica. Complex expressions for oscillatory motions are solved using approximations to confirm their non-existence and onset of convection is established as stationary. Binary nanofluids are found to be much less stable than regular fluids. Higher conductivity of metallic nanofluids makes them less stable as compared to non-metallic nanofluids.
\end{abstract}

Keywords- Binary nanofluid, Brownian motion, Thermophoresis, Chandrasekhar number, Natural convection.

\section{Introduction}

The origin of nanofluids is attributed to the revolutionary idea of suspension of nanoparticles into heat transfer liquids to enhance the thermal conductivity (Choi, 1995). Buongiorno (2006) developed equations for mass, momentum and heat transfer in nanofluids. Since then, a large number of mathematical studies on convective heat transfer in nanofluids became feasible. Tzou (2008) initiated the work on thermal instability of nanofluids using the model given by Buongiorno (2006) and found that addition of nanoparticles make the fluid more unstable. Further, the convection in nanofluids was investigated analytically for various boundaries by Nield and Kuznetsov (2010). Gupta et al. (2013) carried forward their work by extending the nanofluid convection problems under magnetic field. To study the interesting effects of thermal and solutal diffusivities, convection in a binary nanofluid layer was first conducted by Nield and Kuznetsov (2011). The stabilizing impact of magnetic field and rotation on double diffusive convection in nanofluids was established by Gupta et al. (2015) and Sharma et al. (2016), respectively.

Recently, the thermal convection in nanofluids was revisited by Nield and Kuznetsov (2014a, b) with more practical boundary conditions in which the zero nanoparticle flux was considered at the boundaries and absence of oscillatory motions was established. Further, Agarwal (2014) and Chand and Rana (2015) considered nanofluid convection saturating porous medium using revised model under Coriolis and Lorentz forces, respectively. In the present work, we have examined the 
International Journal of Mathematical, Engineering and Management Sciences

Vol. 4, No. 1, 131-138, 2019

https://dx.doi.org/10.33889/IJMEMS.2019.4.1-012

instability of a binary nanofluid layer under magnetic field. The equations for the system are made dimensionless and small disturbances are imposed on the primary flow. Further, method of normal modes is used to get the expression for thermal Rayleigh number. Complex calculations are simplified using valid approximations to study the problem analytically. Effects of solute concentration difference, temperature difference, nanoparticles volume fraction and magnetic field parameter on thermal Rayleigh number are analyzed with stability curves for alumina (non-metal) and copper (metal) nanoparticles with the help of software Mathematica.

\section{Problem Formulation}

Consider a nanofluid layer of width $d$ which is soluted and heated from below. The conservation equations (refer: Gupta et al., 2015) are

$$
\begin{aligned}
& \nabla . \boldsymbol{u}=0 \\
& \rho\left(\frac{\partial \boldsymbol{u}}{\partial t}+\boldsymbol{u} \cdot \nabla \boldsymbol{u}\right)=\mu \nabla^{2} \boldsymbol{u}-\nabla p+\left[\phi \rho_{p}+(1-\phi)\left\{\rho\left(1-\beta_{C}\left(C-C_{0}\right)-\beta_{T}\left(T-T_{0}\right)\right)\right\}\right] \boldsymbol{g}+\frac{\mu_{e}}{4 \pi}(\boldsymbol{H} . \nabla) \boldsymbol{H}, \\
& \rho c\left[\frac{\partial T}{\partial t}+\boldsymbol{u} \cdot \nabla T\right]=\rho_{p} c_{p}\left[D_{B} \nabla T . \nabla \phi+\frac{D_{T}}{T_{0}} \nabla T . \nabla T\right]+k \nabla^{2} T+\rho c D_{T C} \nabla^{2} C \\
& \frac{\partial \phi}{\partial t}+\boldsymbol{u} \cdot \nabla \phi=D_{B} \nabla^{2} \phi+\frac{D_{T}}{T_{0}} \nabla^{2} T \\
& \frac{\partial C}{\partial t}+\boldsymbol{u} \cdot \nabla C=D_{S} \nabla^{2} C+D_{C T} \nabla^{2} T .
\end{aligned}
$$

The Maxwell's equations are:

$$
\begin{aligned}
& \frac{\partial \boldsymbol{H}}{\partial t}+(\boldsymbol{u} . \nabla) \boldsymbol{H}=(\boldsymbol{H} . \nabla) \boldsymbol{u}+\eta \nabla^{2} \boldsymbol{H}, \\
& \nabla . \boldsymbol{H}=0,
\end{aligned}
$$

and conditions for free boundaries are:

$$
\begin{aligned}
& w=0, T=T_{1}, D_{B} \frac{\partial \phi}{\partial z}+\frac{D_{T}}{T_{0}} \frac{\partial T}{\partial z}=0 \quad C=C_{1} \quad \text { at } z=0, \\
& w=0, \quad T=T_{0}, D_{B} \frac{\partial \phi}{\partial z}+\frac{D_{T}}{T_{0}} \frac{\partial T}{\partial z}=0 \quad C=C_{0} \text { at } z=d .
\end{aligned}
$$

Here, $\boldsymbol{u}=(u, v, w) \phi, t, \rho_{p}, D_{B}, D_{T}, \mu,(\rho c)_{f},(\rho c)_{p}, k, D_{S}, \quad \boldsymbol{H}=\left(H_{x}, H_{y}, H_{z}\right), T, p, \mu$, $\beta_{C}, \beta_{T}, g, \rho, c, k, c_{p}, D_{T C}, C, D_{C T}, \eta$ respectively, are the fluid velocity, the particle volume fraction, the time, the particle density, the Brownian coefficient, the thermophoretic coefficient, the fluid viscosity, the fluid heat capacity, the nanoparticles heat capacity, the thermal conductivity of medium, the solutal diffusivity, the magnetic field, the fluid temperature, the pressure, the fluid viscosity, the solute volumetric coefficient, the thermal volumetric coefficient, the gravitational acceleration, the fluid density, the fluid specific heat, the fluid conductivity, the 
International Journal of Mathematical, Engineering and Management Sciences

Vol. 4, No. 1, 131-138, 2019

https://dx.doi.org/10.33889/IJMEMS.2019.4.1-012

nanoparticle specific heat, the Dufour diffusivity, the solute concentration, the Soret diffusivity and the electrical resistivity of the nanofluid. Let us non-dimensionalize variables as:

$(x, y, z) d=(x, y, z), t d^{2}=t \alpha_{f}, \phi \phi_{b}=\phi, \boldsymbol{u} \alpha_{f}=\boldsymbol{u} d, p \mu \alpha_{f}=p d^{2}, \boldsymbol{H} H_{0}=\boldsymbol{H}$

$T\left(T_{1}-T_{0}\right)=\left(T-T_{0}\right), \quad C\left(C_{1}-C_{0}\right)=\left(C-C_{0}\right)$,

where $\alpha_{f}=k / \rho c$ and $\left(0,0, H_{0}\right)$ is applied magnetic field. Eqs. (1)-(9) become:

$\nabla \cdot \boldsymbol{u}=0$,

$\frac{1}{P r_{1}}\left(\frac{\partial \boldsymbol{u}}{\partial t}+\boldsymbol{u} \cdot \nabla \boldsymbol{u}\right)=-\nabla p+\nabla^{2} \boldsymbol{u}-R m \hat{k}+R_{A} T \hat{k}-R n \phi \hat{k}+\frac{R s}{L_{s}} C \hat{k}+Q \frac{P r_{1}}{P r_{2}} \frac{\partial \boldsymbol{H}}{\partial z}$,

$\frac{\partial T}{\partial t}+\boldsymbol{u} . \nabla T=\frac{N_{B}}{L_{e}} \nabla \phi . \nabla T+\frac{N_{A} N_{B}}{L_{e}} \nabla T . \nabla T+N_{T C} \nabla^{2} C+\nabla^{2} T$,

$\frac{\partial C}{\partial t}+\boldsymbol{u} \cdot \nabla C=\frac{1}{L_{s}} \nabla^{2} C+N_{C T} \nabla^{2} T$,

$\frac{\partial \phi}{\partial t}+\boldsymbol{u} . \nabla \phi=\frac{1}{L_{e}} \nabla^{2} \phi+\frac{N_{A}}{L_{e}} \nabla^{2} T$,

$\frac{\partial \boldsymbol{H}}{\partial t}+(\boldsymbol{u} . \nabla) \boldsymbol{H}=(\boldsymbol{H} . \nabla) \boldsymbol{u}+\frac{P r_{1}}{P r_{2}} \nabla^{2} \boldsymbol{H}$,

$\nabla . \boldsymbol{H}=0$,

$w=0, \frac{\partial \phi}{\partial z}+N_{A} \frac{\partial T}{\partial z}=0, \quad C=T=1, \quad$ at $\quad z=0$,

$w=0$,

$\frac{\partial \phi}{\partial z}+N_{A} \frac{\partial T}{\partial z}=$

$C=T=0 \quad$,

$z=1$,

where dimensionless numbers are: Prandtl number $\operatorname{Pr}_{1}=\mu / \rho \alpha_{f}$; Magnetic Prandtl number $P r_{2}=\mu / \rho \eta$; fluid Lewis number $L_{e}=\alpha_{f} / D_{B} ;$ solute Lewis number $L_{s}=\alpha_{f} / D_{S}$; Chandrasekhar number $Q=\mu_{e} H_{0}^{2} d^{2} / 4 \pi v \rho \eta$; diffusivity ratio $N_{A}=D_{T}\left(T_{1}-T_{0}\right) / D_{B} T_{0} \phi_{b}$; density increment $N_{B}=(\rho C)_{\mathrm{P}} \phi_{b} / \rho C$; Rayleigh number $R_{A}=\rho \boldsymbol{g} \beta_{T} d^{3}\left(T_{1}-T_{0}\right) / \mu \alpha_{f} ;$ solutal Rayleigh number $R s=\rho g \beta_{C} d^{3}\left(\mathrm{C}_{1}-C_{0}\right) / \mu D_{S}$; basic-density Rayleigh number, $R m=\rho \boldsymbol{g} d^{3} / \mu \alpha_{f}$; nanoparticle Rayleigh number $R n=\left(\rho_{p}-\rho\right) \phi_{b} \boldsymbol{g} d^{3} / \mu \alpha_{f}$; Dufour parameter $N_{T C}=D_{T C}\left(C_{1}-C_{0}\right) / \alpha_{f}\left(T_{1}-T_{0}\right) ; \quad$ Soret parameter $N_{C T}=D_{C T}\left(T_{1}-T_{0}\right) / \alpha_{f}\left(C_{1}-C_{0}\right)$.

\section{Method of Analysis}

Initially, system is at rest and all the parameters vary in horizontal direction only. Eqs. (11)-(19) become 
International Journal of Mathematical, Engineering and Management Sciences

Vol. 4, No. 1, 131-138, 2019

https://dx.doi.org/10.33889/IJMEMS.2019.4.1-012

$\frac{d p}{d z}+R_{M}-R_{T} T+R_{N} \phi-\frac{R_{S}}{L_{S}} C=0$,

$\frac{d^{2} T}{d z^{2}}+\frac{N_{B}}{L_{N}} \frac{d \phi}{d z} \frac{d T}{d z}+\frac{N_{A} N_{B}}{L_{N}}\left(\frac{d T}{d z}\right)^{2}+N_{T C} \frac{d^{2} C}{d z^{2}}=0$,

$\frac{d^{2} \phi}{d z^{2}}+N_{A} \frac{d^{2} T}{d z^{2}}=0$

$\frac{1}{L_{S}} \frac{d^{2} C}{d z^{2}}+N_{C T} \frac{d^{2} T}{d z^{2}}=0$.

The solution of Eqs. (21)-(24) satisfying boundary conditions (18) and (19) give

$\frac{d \phi}{d z}=N_{A} z, T=1-z, C=1-z$.

Note that there is no restriction on the value of nanofluid Lewis number to find basic solution. Previously, we had to assume the value of nanofluid Lewis number to be large for analytical results. Thus, revised model gives more generalized basic solution.

Let us apply small disturbances $(\tilde{\boldsymbol{u}}, \tilde{p}, \tilde{\boldsymbol{H}}, \tilde{T}, \tilde{\boldsymbol{C}}, \tilde{\phi})$ on Eqs. (25), we get

$\nabla . \tilde{\boldsymbol{u}}=0$,

$\frac{1}{P r_{1}}\left(\frac{\partial \tilde{\boldsymbol{u}}}{\partial t}+\tilde{\boldsymbol{u}} \cdot \nabla \tilde{\boldsymbol{u}}\right)=-\nabla \tilde{p}+\nabla^{2} \tilde{\boldsymbol{u}}+R_{A} \tilde{T} \hat{k}-R n \tilde{\phi} \hat{k}+\frac{R s}{L_{s}} \tilde{C} \hat{k}+Q \frac{P r_{1}}{P r_{2}} \frac{\partial \tilde{\boldsymbol{H}}}{\partial z}$,

$\frac{\partial \tilde{T}}{\partial t}-\tilde{w}+\frac{N_{B}}{L_{e}}\left(\frac{\partial \tilde{\phi}}{\partial z}+N_{A} \frac{\partial \tilde{T}}{\partial z}\right)=N_{T C} \nabla^{2} \tilde{C}+\nabla^{2} \tilde{T}$,

$\frac{\partial \tilde{C}}{\partial t}-\tilde{w}=\frac{1}{L_{s}} \nabla^{2} \tilde{C}+N_{C T} \nabla^{2} \tilde{T}$,

$\frac{\partial \tilde{\phi}}{\partial t}+N_{A} \tilde{w}=\frac{1}{L_{e}} \nabla^{2} \tilde{\phi}+\frac{N_{A}}{L_{e}} \nabla^{2} \tilde{T}$,

$\frac{\partial \tilde{\boldsymbol{H}}}{\partial t}=\frac{\partial \tilde{w}}{\partial z} \hat{k}+\frac{\operatorname{Pr}_{1}}{\operatorname{Pr}_{2}} \nabla^{2} \tilde{\boldsymbol{H}}$,

$\nabla . \tilde{\boldsymbol{H}}=0$,

Following the technique of normal modes (superposition of basic modes), we get an eigenvalue equation.

\section{Analytical Results and Discussions 4.1 Non-Oscillatory Convection}

Let growth rate $s=0$ for stationary convection in eigenvalue equation, this gives $R_{A}$ as 
International Journal of Mathematical, Engineering and Management Sciences

Vol. 4, No. 1, 131-138, 2019

https://dx.doi.org/10.33889/IJMEMS.2019.4.1-012

$R_{A}=\frac{1}{\left(1-L_{s} N_{T C}\right)}\left\{\left(\frac{Z^{3}+Z \pi^{2} Q}{a^{2}}-R n N_{A} L_{e}\right)\left(1-N_{C T} N_{T C} L_{s}\right)-R s\left(1-N_{C T}\right)\right\}-R n N_{A}$.

where $Z=\pi^{2}+a^{2}$. Clearly, magnetic field stabilizes the system significantly and the occurrence of nanoparticles hastens the onset of convection. In the absence of Soret parameter, solute Rayleigh number destabilizes the system. Also, note that existence of nanoparticles does not influence the value of critical wave number.

\subsection{Oscillatory Convection}

For oscillatory convection; $s=i \omega$. Complex expressions are obtained from eigenvalue equation which are analyzed by substituting $L_{s}=1, \operatorname{Pr}, L_{e} \rightarrow \infty$ and $N_{C T}, N_{T C} \rightarrow 0$, we get all the values of $\omega^{2}$ as negative. Thus absence of oscillatory motions is established.

\section{Numerical Results and Discussions}

The influence of solute concentration difference, temperature difference and nanoparticle volume fraction don't affect the stability of the layer directly as they enter in the system via different non-dimensional numbers and hence couldn't be studied analytically. Keeping in mind the importance of the influence of parameters on instability of the layer, numerical computations are accomplished for metal (copper) and non-metal (alumina) using the software Mathematica. Figures 1-5 show the stability curves for values of nanofluid parameters as: copper $R n=0.1, N_{A}=0.5$; alumina; $R n=0.03, \quad N_{A}=5$. Let us fix other parameters as: $L_{s}=2 ; \quad N_{T C}=0.01 ; \quad N_{C T}=2$; $R s=50 ; \quad Q=100 ;$ for water based nanofluid: $L_{e}=5000$.

Figure 1 shows the destabilizing influence of temperature difference on the boundaries (appears in the parameters $N_{A}, N_{C T}, N_{T C}$ ) and alumina nanoparticles are observed to have more impact on instability of the system than copper because of its lower thermal conductivity. The stabilizing influence of solute concentration difference on the boundaries of the layer (appears in the parameters $R s, N_{C T}, N_{T C}$ ) is analyzed in Figure 2. It is worth mentioning that the stabilizing influence is same for different physical properties of nanoparticles. 
International Journal of Mathematical, Engineering and Management Sciences

Vol. 4, No. 1, 131-138, 2019

https://dx.doi.org/10.33889/IJMEMS.2019.4.1-012

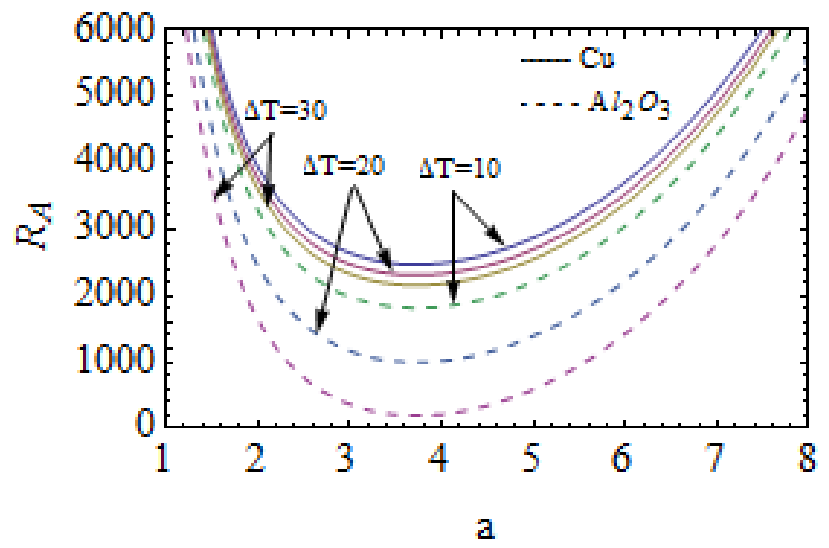

Figure 1. Impact of temperature difference

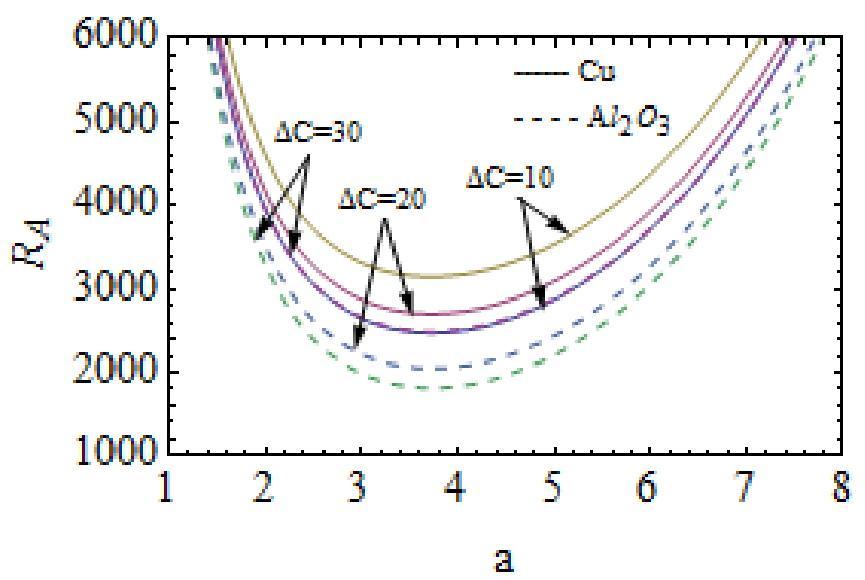

Figure 2. Impact of solute concentration difference

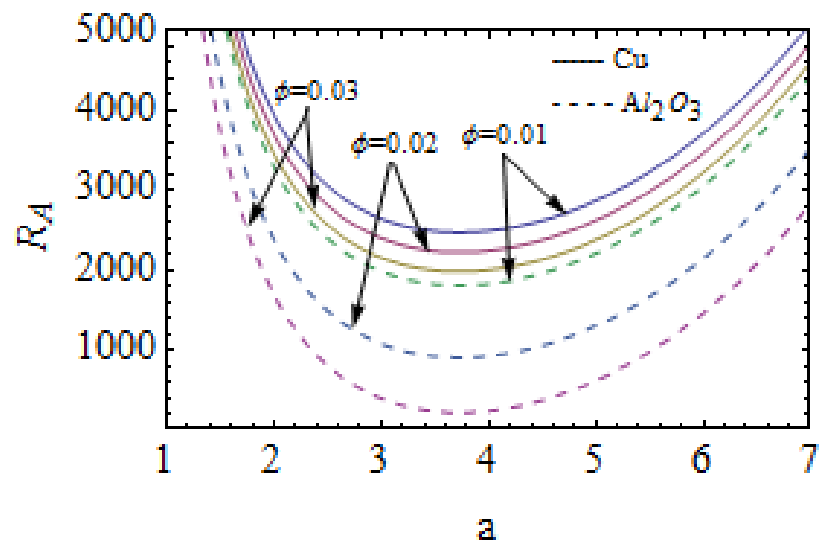

Figure 3. Impact of nanoparticle volume fraction 
International Journal of Mathematical, Engineering and Management Sciences

Vol. 4, No. 1, 131-138, 2019

https://dx.doi.org/10.33889/IJMEMS.2019.4.1-012

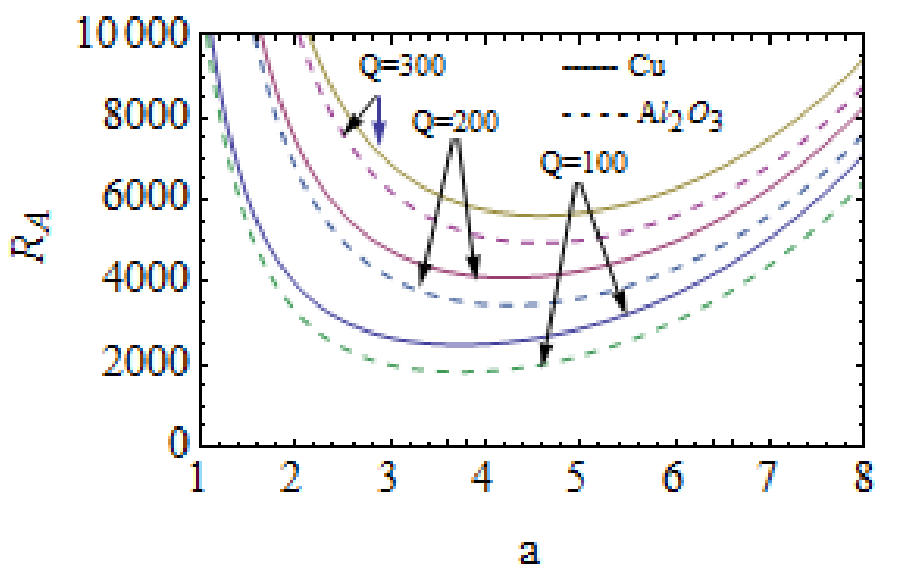

Figure 4. Impact of Chandrasekhar number

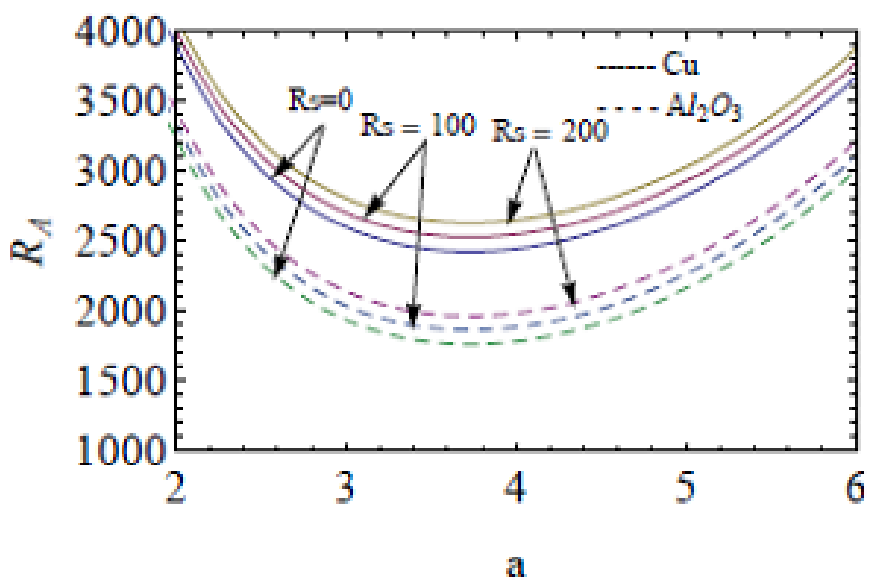

Figure 5. Impact of solute Rayleigh number

Figure 3 establishes the strong destabilizing influence of nanoparticles (enters through the parameters $R n, N_{A}$ ) on the stability which is more pronounced for alumina than copper. The magnetic field parameter delays the onset of convection for metallic and non-metallic nanofluids as observed in Figure 4. The impact of solute Rayleigh number is also found to be stabilizing for metallic and non-metallic nanoparticles as shown in Figure 5.

Here, it is worthwhile to mention that in all the Figures. (1)-(5), alumina (non-metal) nanoparticles destabilize the system more than copper (metal). The reason lies in the fact that higher conductivity of metals leads to higher values of corresponding non-dimensional number (diffusivity ratio) which decreases the value of Rayleigh number significantly, making metals less stable than non-metals in water based nanofluids. 
International Journal of Mathematical, Engineering and Management Sciences

Vol. 4, No. 1, 131-138, 2019

https://dx.doi.org/10.33889/IJMEMS.2019.4.1-012

\section{Conclusions}

The thermosolutal instability of a nanofluid layer under magnetic field is examined using conservations equations for the system. The model used to analyze the problem is revised under more realistic boundary conditions of zero nanoparticle flux. Non-existence of oscillatory motions is established leading to stationary mode of instability in the layer. Addition of nanoparticles increases the instability of the system significantly. Alumina nanoparticles quicken the convection at the higher rate as compared to copper. The temperature difference, solute concentration difference and nanoparticle volume fraction destabilize the system while magnetic field parameter delays the onset of convection in the layer. Solute Rayleigh number stabilizes the system while its influence is found to be destabilizing in the absence of solutal effects.

\section{Conflict of Interest}

The authors confirm that there is no conflict of interest to declare for this publication.

\section{Acknowledgement}

The research work presented has been supported by grants to second and third authors from Council of Scientific and Industrial Research, New Delhi-110012, India in the form of Research and Development Project [Ref. No: 25(0247)/15/EMR-II].

\section{References}

Agarwal, S. (2014). Natural convection in a nanofluid-saturated rotating porous layer: a more realistic approach. Transport in Porous Media, 104(3), 581-592.

Buongiorno, J. (2006). Convective transport in nanofluids. ASME Journal of Heat Transfer, 128(3), 240-250.

Chand, R., \& Rana G. C. (2015). Magneto convection in a layer of nanofluid in porous medium-a more realistic approach, Journal of Nanofluids, 4(2), 196-202.

Choi, S. (1995). Enhancing thermal conductivity of fluids with nanoparticles, in Development and Applications of Non-Newtonian Flows, D. A. Siginer and H. P. Wang, Eds., 66, ASME FED-231/MD, 99-105.

Gupta, U., Ahuja J., \& Wanchoo, R. K. (2013). Magneto convection in a nanofluid layer. International Journal Heat and Mass Transfer, 64, 1163-1171.

Gupta, U., Sharma J., \& Sharma V. (2015). Instability of binary nanofluid with magnetic field. Applied Mathematics and Mechanics, 36(6), 693-706.

Nield, D. A., \& Kuznetsov A. V. (2011). The onset of double-diffusive convection in a nanofluid layer. International Journal of Heat and Fluid Flow, 32(4), 771-776.

Nield, D. A., \& Kuznetsov, A. V. (2014a). Thermal instability in a porous medium layer saturated by a nanofluid: a revised model. International Journal of Heat and Mass Transfer, 68, 211-214.

Nield, D. A., \& Kuznetsov, A. V. (2014b). The onset of convection in a horizontal nanofluid layer of finite depth: A revised model. International Journal of Heat and Mass Transfer, 77, 915-918.

Nield, D. A., \& Kuznetsov, A. V. (2010). The onset of convection in a horizontal nanofluid layer of finite depth. European Journal of Mechanics-B/Fluids, 29(3), 217-223.

Sharma, J., Gupta U., \& Wanchoo, R. K. (2017). Numerical Study on Binary Nanofluid Convection in a Rotating Porous Layer. Differential Equations and Dynamical Systems, 25(2), 239-249.

Tzou, D. Y. (2008) Thermal instability of nanofluids in natural convection. International Journal of Heat and Mass Transfer, 51, 2967-2979. 